\title{
Fast Initial State Assessment for State Estimator using Optimally Located Phasor Measurement Units
}

\author{
M.T.L.Gayatri \\ Assistant Professor, EEE \\ Department, Sreenidhi Institute \\ of Science and Technology, \\ Ghatkesar, Hyderabad, Andhra \\ Pradesh, INDIA
}

\author{
A.V.R.S.Sarma \\ Professor, EE Department, \\ College of Engineering, \\ Osmania University, \\ Hyderabad, Andhra Pradesh, \\ INDIA
}

\author{
Chandragiri Radha \\ Charan \\ Assistant Professor, EEE \\ Department, JNTUH, College of \\ Engineering, Kondagattu, \\ Karimnagar (Dist.), Andhra \\ Pradesh, INDIA
}

\begin{abstract}
Complex power systems need better observability and visualization capabilities to handle critical situations effectively. Synchro-phasor measurements provide intra second visibility to power system dynamics and enable faster control actions. A Phasor Measurement Unit (PMU) measures the voltage and current phasors (angle and magnitude) at a bus in a power system. In the literature, many methods have been reported for determining a measurement set for the allocation of PMU's. Most of these methods use conventional optimization techniques involving more mathematical equations, which are generally time-consuming from a computation point of view, especially for large systems under emergency conditions. The operator has to take instantaneous measurements for the quick state estimation. In this paper a simplified approach has been proposed for placing the measuring devices (PMU's) which involves graph theoretical approach only .A linear algorithm is used to minimize the number of PMUs to monitor the entire system by modeling the system with topological observation theory. A relationship was developed between phasors where PMU's are located and unknown phasors of the system. The results can be used as initial assessment for the state estimator. The algorithm was tested on IEEE-14 Bus System.
\end{abstract}

\section{General Terms}

Phasor Measurement Units, Graph Theory

\section{Keywords}

Phasor Measurement Unit, Dominant set, Systemphasors, Synchrophasors, Universal Time Coordinated, Phasor Data Concetrator.

\section{INTRODUCTION}

system is dynamic, the operating conditions are changing continuously and results in frequent changes of system topology (network interconnections). This is because of uncertainties in the rapid growth in the demand of electricity, the requirement of power system components to be operated closer to their designed limits and high penetration of renewable energy sources. So continuous real time monitoring of the system is essential .For decades, traditional SCADA measurements have been providing information on bus voltages, line, generator and transformer flows (MW, MVAR and Amperes), transformer taps and breaker status as well as other system parameters viz, frequency and weather data. These measurements are typically taken once every 4 or 10 seconds offering a steady state view of the power system behavior. However, for monitoring and control of such large grid only steady state information may not be sufficient. A phasor measurement unit (PMU) [11] is a device used to measure the phasor values of currents and voltages. The phasors are measured by sampling the sinusoidal waveform at appropriate time intervals and over a sufficient time period. The phase angle of the measured phasors is referenced to a coordinated universal time (UTC). Thus, the phasors are synchronized with respect to a common time reference and hence they are called synchro-phasors.PMU measurements are taken at high speed typically 25 or 50 observations per second - compared to one every 4 to 10 seconds using conventional technology. Each measurement is time-stamped according to a common time reference. Time stamping allows synchrophasors[11] from different locations to be timealigned (or synchronized) providing combined and comprehensive view of the entire grid. Synchrophasors enable a better indication of grid stress, and can be used to trigger corrective actions to maintain reliability. Synchrophasor data could be used to allow power flow up to a line's dynamic limit. Therefore this technology has the potential to change the economics of power delivery by allowing optimum level of power flow over existing lines.

The concept of phasors is very useful in describing the behavior of a power system in its steady state as well as in transient state. Although phasors are better understood as representing a steady state sinusoidal waveform, phasor measurements can be used to derive a variety of information about the transient state of a power system [1]. Phasor measurement technologies have undergone many changes, first with the advent of digital electronic systems and then with the increasing need for synchronized phasor measurements at different points of large power systems. Synchrophasor Measurement technology provides dynamic view of the grid along with information like load angle between different locations of the grid.

\section{SYSTEM OBSERVABILITY}

A power system with a specified measurement set is said to be observable if an estimate of the state vector of bus voltage magnitudes and angles can be made. Static state assessment is the process of determining the bus voltage magnitudes and angles from a set of measurements which ordinarily consists of bus voltage magnitudes, real and reactive line flow powers and real and reactive bus injection powers. A fundamental question with regard to the measurements on the network is whether it is possible to determine the bus voltage magnitudes and angles throughout the entire network from them. If this is possible, the network is said to be observable. A power 
system is called completely observable when all of its states (i.e. voltage magnitudes and phase angles) can be uniquely determined based on the available measurements. The observability of a system can be assessed by considering the topology of the network and the types and locations of the measurements. According to Clements et al,[1], two different observability concepts can be defined as approaches to observability analysis:

Topological: The system is taken as observable if it is possible to construct a measurement tree that spans the whole network.

Numerical: Various numerical techniques (Genetic Algorithm, Artificial Intelligence etc.) are there to check the observability of the network. Numerical observability implies topological observability, but not the converse.

\section{Observability Rules}

1. Any bus (or alternatively called, nodes, vertices) with a PMU is observed. So is (are) the directly adjacent bus (es).

2. Any line (or alternatively called, edges) between two observed buses is observed.

3. Any bus that is incident to an observed line is observed.

4. If a bus is incident to a total of $k>1$ lines and if $k-1$ of those lines are observed, then all $\mathrm{k}$ of these lines are observed. This rule is based on Kirchhoff's current law.

\section{OPTIMAL ALLOCATION OF PMU'S 3.1 Optimality and Node to Node Incidence Matrix}

It is not possible to locate PMUs at each and every bus of the network. Complete Observability[7] of the system should be achieved with minimum number of PMUs used. Then the PMUs are said to be optimally located.

Linear algorithm[9] is the algorithm based on spanning of the network measured in terms of incidence of nodes in order to achieve the total observability of the system

The topological information of a power system can be stored in Node-Node Incidence Matrix [9] for computer analysis. The rule is given below if node $i$ is incident to node $j$, then $\mathrm{Aij}=1, \mathrm{i} \neq \mathrm{j}$ otherwise $=0$. Normally $\mathrm{A}$ is a large sparse matrix.

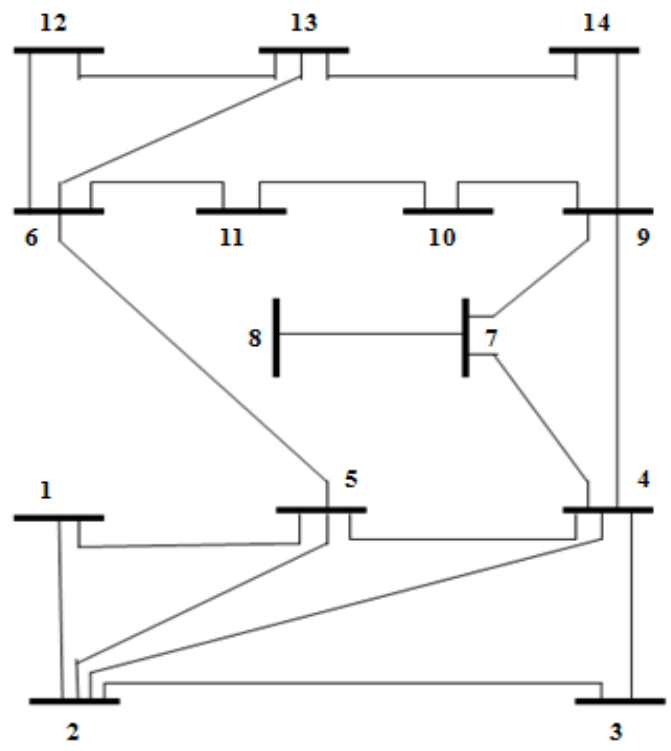

Fig.1. IEEE- 14 Bus System
Table 2: IEEE-14 bus system Node - Node Incidence Matrix, "A"

$\left[\begin{array}{llllllllllllll}0 & 1 & 0 & 0 & 1 & 0 & 0 & 0 & 0 & 0 & 0 & 0 & 0 & 0 \\ 1 & 0 & 1 & 1 & 1 & 0 & 0 & 0 & 0 & 0 & 0 & 0 & 0 & 0 \\ 0 & 1 & 0 & 1 & 0 & 0 & 0 & 0 & 0 & 0 & 0 & 0 & 0 & 0 \\ 0 & 1 & 1 & 0 & 1 & 0 & 1 & 0 & 1 & 0 & 0 & 0 & 0 & 0 \\ 1 & 1 & 0 & 1 & 0 & 1 & 0 & 0 & 0 & 0 & 0 & 0 & 0 & 0 \\ 0 & 0 & 0 & 0 & 1 & 0 & 0 & 0 & 0 & 0 & 1 & 1 & 1 & 0 \\ 0 & 0 & 0 & 1 & 0 & 0 & 0 & 1 & 1 & 0 & 0 & 0 & 0 & 0 \\ 0 & 0 & 0 & 0 & 0 & 0 & 1 & 0 & 0 & 0 & 0 & 0 & 0 & 0 \\ 0 & 0 & 0 & 1 & 0 & 0 & 1 & 0 & 0 & 1 & 0 & 0 & 0 & 1 \\ 0 & 0 & 0 & 0 & 0 & 0 & 0 & 0 & 1 & 0 & 1 & 0 & 0 & 0 \\ 0 & 0 & 0 & 0 & 0 & 1 & 0 & 0 & 0 & 1 & 0 & 0 & 0 & 0 \\ 0 & 0 & 0 & 0 & 0 & 1 & 0 & 0 & 0 & 0 & 0 & 0 & 1 & 0 \\ 0 & 0 & 0 & 0 & 0 & 1 & 0 & 0 & 0 & 0 & 0 & 1 & 0 & 1 \\ 0 & 0 & 0 & 0 & 0 & 0 & 0 & 0 & 1 & 0 & 0 & 0 & 1 & 0 \\ & & & & & & & & & & & & & \end{array}\right]$

\subsection{Dominant Set}

Degree of a vertex indicates number of other buses that are incident to that vertex.Dominant set[9] is the set of buses where PMU's are mounted on. In 2002, Haynes et al. [7], mathematically proved that, for a tree having $\mathrm{k}$ vertices of degree at least 3, the "power dominating number" has been minimized where $\mathrm{n}$ is the total number of vertices. Above equations give the upper and lower bounds for the power dominating number[9]. It is easy to find out that there are 7 nodes with degree 3 or node in S-set has degree at least 3 (buses with at least 3 lines) for the system. The corresponding nodes are 2, 4,5,6,7,9,13 (degree more than are equal to 3 )

$\mathrm{k}=7, \mathrm{n}=14$.

$$
\begin{aligned}
& \lambda_{p}(T) \geq \frac{k+2}{3} \\
& \lambda_{p}(T) \leq \frac{n}{3}
\end{aligned}
$$

So, the bounds of number of PMUs needed are

$$
\begin{aligned}
& \lambda_{p}(T) \geq \frac{7+2}{3}=3 \\
& \lambda,(T) \leq \frac{14}{3}=4.67 \rightarrow 4
\end{aligned}
$$

That is, the only possible values for dominant set " $S$ " [7]are 3 and 4 . The measurement of the algorithm having known the bounds, to find out the minimum number of PMU's, and the dominating set $\mathrm{S}$.The basic idea of this algorithm[9] is to test all possible node combinations by the observation rules, until one combination is found to be able to observe" all the system. Test for a combination (with bound constrains) is known as a measurement. Obviously, for the IEEE 14-bus system, the maximum number of measurements is ${ }_{4} \mathrm{C}+{ }_{4}^{14} \mathrm{C}$

Because the S-set may contain either 3 or 4 nodes. Taking advantage of the observation rule 4 [7], 7 nodes are identified with degree 3.There are 7 nodes have degree at least 3 (buses with at least 3 lines), more. The bounds of number of PMUs needed can be found from the maximum number of measurements for the 14- bus system[9] is ${ }_{3}^{7} \mathrm{C}+{ }_{4}^{7} \mathrm{C}=70$ 


\subsection{Linear Algorithm}

1. Read in Node-Node incidence matrix A. All buses (nodes) are in the graph set $\mathrm{G}$.

2. Determine the bounds of dominant set $S$

3. Loop starts from the lower bound to the upper bound

4. Generate a node combination, e.g., $\{2,4,5\}$. These nodes are mounted with PMUs, thus observed. Save them in array C.

5. Find out all nodes adjacent to the nodes in selected combination. Save them in array $\mathrm{C}$

a) If $\mathrm{C}=\mathrm{G}$, output the node combination as "Dominant set" go to step 7

b) If $\mathrm{C}$ and $\mathrm{G}$ are unequal go to next step ' 6 '

6. Find out all nodes not in $\mathrm{C}$.

a). Pick up such a node j, use observation rule 4 to judge if it is observed.

b). If yes, put $\mathrm{j}$ in $\mathrm{C}$, and pick up another node and check

c). If all "not-in-C" nodes have been checked, compare $\mathrm{C}$ to the whole set $\mathrm{G}$.

- If $\mathrm{C}=\mathrm{G}$, output the node combination in a). That is the $\mathrm{S}$ set. go to step7.

- If C is not equal to $\mathrm{G}$, go to step4 to generate another node combination

7. End.

\subsection{Flow Chart}

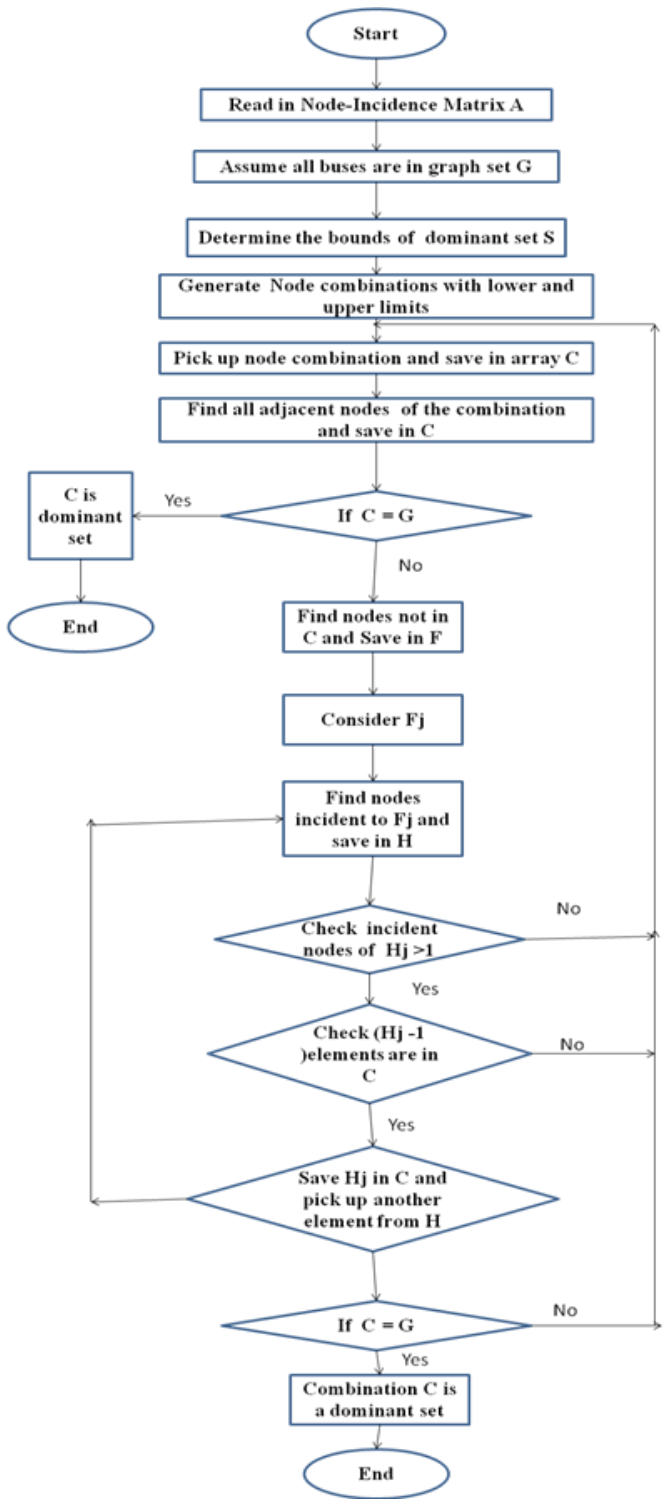

\subsection{System Phasors}

From the placement studies carried in chapter 3, the dominant set with minimum number of PMUs is the optimal choice for the placement of PMUs. For the IEEE-14 Bus test case , dominant sets with minimum number of PMU's are [ 26 7], [4 6 7],[5 6 7].Among these sets any one set like [ $\left.\begin{array}{lll}2 & 6 & 7\end{array}\right]$ is considered for the placement of PMUs. The voltage and current phasors of these buses are considered as available system phasors. It is assumed that only three phasors namely, V2, V6, V7, positive sequence voltage phasors are available. The remaining $\mathrm{V}_{1}, \mathrm{~V}_{3}, \mathrm{~V}_{4}, \mathrm{~V}_{5}, \mathrm{~V}_{8}, \mathrm{~V}_{9}, \mathrm{~V}_{10}, \mathrm{~V}_{11}, \mathrm{~V}_{12}, \mathrm{~V}_{13}, \mathrm{~V}_{14}$ bus voltages need to be determined. Similarly available current phasors are $\mathrm{I}_{2}, \mathrm{I}_{6}, \mathrm{I}_{7}$, and Other unknown current phasors $\mathrm{I}_{1}, \mathrm{I}_{3}, \mathrm{I}_{4}, \mathrm{I}_{5}, \mathrm{I}_{8}, \mathrm{I}_{9}, \mathrm{I}_{10}, \mathrm{I}_{11}, \mathrm{I}_{12}, \mathrm{I}_{13}, \mathrm{I}_{14}$ need to be determined.

Let $\mathrm{V}_{\mathrm{p}}$ be a vector of measured voltages in polar form at the 3 PMU buses,and let $\mathrm{V}_{\mathrm{n}}$ be a vector of unmeasured voltages in polar form at the remaining 11 buses.

Note: Bus 1 is slack bus.

$\left[\mathrm{V}_{\mathrm{p}}\right]_{3 \times 1}=$ PMU measured voltage quantities.

$\left[\mathrm{V}_{\mathrm{n}}\right]_{11 \times 1}=$ unmeasured voltage quantities

Similarly

$\left[\mathrm{I}_{\mathrm{p}}\right]_{3 \times 1}=\mathrm{PMU}$ measured current quantities

$\left[\mathrm{I}_{\mathrm{n}}\right]_{11 \times 1}=$ unmeasured current quantities

From the relation

$$
[\mathrm{I}]=[\mathrm{Y}] *[\mathrm{~V}]
$$

Vector I is divided

into $I_{p}$ and $I_{n}$ where $I_{p}$ is the vector of measured quantities, $I_{n}$ is the vector of unmeasured quantities.

The above equation can be rewritten as $\left[\begin{array}{c}\mathbf{I} \mathbf{p} \\ \mathbf{I n}\end{array}\right]=$

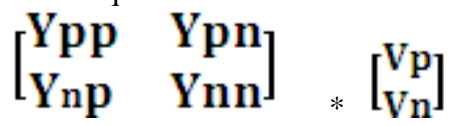

Where $\left[\mathrm{Y}_{\mathrm{pp}}\right],\left[\mathrm{Y}_{\mathrm{pn}}\right],\left[\mathrm{Y}_{\mathrm{np}}\right],\left[\mathrm{Y}_{\mathrm{nn}}\right]$ are partitioned portions of network Y-bus matrix with and without PMU's (p- pmu, nother than pmu)

Consider $\mathrm{I}_{\mathrm{n}}=\mathrm{Y}_{\mathrm{np}} \mathrm{V}_{\mathrm{p}}+\mathrm{Y}_{\mathrm{nn}} \mathrm{V}_{\mathrm{n}}$

$\mathrm{Y}_{\mathrm{np}}, \mathrm{Y}_{\mathrm{nn}}$ are available sub matrices from matrix $\mathrm{Y}_{\text {bus }}$ i.e. $\mathrm{Y}$ $\mathrm{I}_{\mathrm{n}}$ can be written as

$I_{n}=\frac{P-j Q}{V_{n}^{*}}$

Where $\mathrm{P}=\mathrm{P}_{\mathrm{g}}-\mathrm{P}_{1}$ and $\mathrm{Q}=\mathrm{Q}_{\mathrm{g}}$ -

$\mathrm{Q}_{\mathrm{l}} \quad$ are the net power injections at unmeasured nodes.

$\mathrm{I}_{\mathrm{n}}=\mathrm{Y}_{\ln } * \mathrm{~V}_{\mathrm{n}}$ where $\mathrm{Y}_{\ln }$ is primitive load admittance matrix Equating above 2 relations for $\mathrm{I}_{\mathrm{n}}$

$\mathrm{Y}_{\mathrm{ln}} * \mathrm{~V}_{\mathrm{n}}=[\mathrm{P}-\mathrm{j} \mathrm{Q}] / \mathrm{V}_{\mathrm{n}}^{*}$

$\mathrm{Y}_{\ln }=[\mathrm{P}-\mathrm{j} \mathrm{Q}] /\left|\mathrm{V}_{\mathrm{n}}\right|^{2}$

Where Yln matrix dimension is $\mathrm{nx} 1$

Assume $\mathrm{Vn}=(1+\mathrm{j} 0) \mathrm{pu}$

$\mathrm{Y}_{\mathrm{ln}}=\mathrm{P}-\mathrm{jQ}$

$\mathrm{I}_{\mathrm{n}}=\mathrm{Y}_{\mathrm{np}} \mathrm{V}_{\mathrm{p}}+\mathrm{Y}_{\mathrm{nn}} \mathrm{V}_{\mathrm{n}}$

Substituting for $\mathrm{I}_{\mathrm{n}}=\mathrm{Y}_{\ln } * \mathrm{~V}_{\mathrm{n}}$

$\mathrm{Y}_{\mathrm{ln}} * \mathrm{~V}_{\mathrm{n}}=\mathrm{Y}_{\mathrm{np}} \mathrm{V}_{\mathrm{p}}+\mathrm{Y}_{\mathrm{nn}} \mathrm{V}_{\mathrm{n}}$

$\left(\mathrm{Y}_{\mathrm{ln}}-\mathrm{Y}_{\mathrm{nn}}\right) * \mathrm{~V}_{\mathrm{n}}=\mathrm{Y}_{\mathrm{np}} \mathrm{V}_{\mathrm{p}}$

$\mathrm{V}_{\mathrm{n}}=\left[\operatorname{inv}\left(\mathrm{Y}_{\mathrm{ln}}-\mathrm{Y}_{\mathrm{nn}}\right)\right] * \mathrm{Y}_{\mathrm{np}} * \mathrm{~V}_{\mathrm{p}}$ 
From the above equation $\mathrm{Vn}$, the vector of unmeasured voltages can be calculated i.e. If the phasors $\mathrm{Vp}$ are measured by PMU's that are optimally placed, unknown phasor $\mathrm{Vn}$ can be calculated with the help of following formula (assuming $\mathrm{Vn}$ as $(1+\mathrm{j} 0) \mathrm{pu})$

$\mathrm{V}_{\mathrm{n}}=\left[\operatorname{inv}\left(\mathrm{Y}_{\mathrm{nn}}-\mathrm{Y}_{\mathrm{nn}}\right)\right] * \mathrm{Y}_{\mathrm{np}} * \mathrm{~V}_{\mathrm{p}}$

Initial state assement can be made with the help of above formula and can be given to state estimator. Fast converge can be easily achieved as the initial assessment is closer to the final solution.

\section{RESULTS}

Dominant sets with minimum number of PMUs required are obtained as three for IEEE-14 bus system.

$$
\begin{array}{lll}
2 & 6 & 7 \\
4 & 6 & 7 \\
5 & 6 & 7
\end{array}
$$

\begin{tabular}{|c|c|c|}
\hline Bus no & $\begin{array}{c}\text { Bus Voltages } \\
\text { (Load Flow results) }\end{array}$ & $\begin{array}{c}\text { Bus Voltages using } \\
\text { PMUs }\end{array}$ \\
\hline 2 & 1.0450 & 1.0450 \\
\hline 6 & 1.0700 & 1.0700 \\
\hline 7 & 1.0457 & 1.0457 \\
\hline 1 & 1.0600 & 1.0485 \\
\hline 3 & 1.0100 & 0.9918 \\
\hline 4 & 1.0132 & 0.9881 \\
\hline 5 & 1.0166 & 0.9925 \\
\hline 8 & 1.0800 & 1.0385 \\
\hline 9 & 1.0305 & 0.9858 \\
\hline 10 & 1.0299 & 0.9841 \\
\hline 11 & 1.0461 & 0.9981 \\
\hline 12 & 1.0533 & 1.0030 \\
\hline 13 & 1.0466 & 0.9968 \\
\hline 14 & 1.0193 & 0.9725 \\
\hline
\end{tabular}

Table 3: Voltage magnitudes comparison of IEEE- 14 Bus system

\begin{tabular}{|c|c|c|}
\hline $\begin{array}{c}\text { Bus } \\
\text { number }\end{array}$ & $\begin{array}{c}\text { Bus Angles } \\
\text { (Load Flow results) }\end{array}$ & $\begin{array}{l}\text { Bus Angles using } \\
\text { PMU's }\end{array}$ \\
\hline 2 & -4.9891 & -4.9891 \\
\hline 6 & -14.4469 & -14.4469 \\
\hline 7 & -13.2368 & -13.2368 \\
\hline 1 & 0 & 0.9073 \\
\hline 3 & -12.7492 & -12.6749 \\
\hline 4 & -10.2420 & -9.9885 \\
\hline 5 & -8.7601 & -8.4014 \\
\hline 8 & -13.2368 & -13.4915 \\
\hline 9 & -14.8201 & -15.0878 \\
\hline 10 & -15.0360 & -15.2877 \\
\hline 11 & -14.8581 & -15.0264 \\
\hline 12 & -15.2973 & -15.4364 \\
\hline 13 & -15.3313 & -15.4845 \\
\hline 14 & -16.0717 & -16.3291 \\
\hline
\end{tabular}

Table 4

Bus Angles comparison of IEEE- 14 Bus systems

\section{CONCLUSIONS}

PMU measures the voltages and angles at the buses w.r.to a reference at the rate of 60 samples/second. A software program has been developed for optimal location of PMU units. It is tested on IEEE-14 bus systems. There are several combinations for optimal placement of PMU's so that the entire system is completely observable. By observing the voltages at theses buses it is possible to find the voltages at other buses. In case of IEEE- 14 bus $\{267\}$ is the dominant set. From the voltages measured at optimally located PMU's and load data other bus voltages including slack bus are determined. This result can be used as initial values for the state estimator for on-line monitoring and control.

\section{REFERENCES}

[1] K.A Clements, "Observability Methods and Optimal Meter Placement", Electrical Power and Energy Systems, Vol 12, No. 2, April 1990.

[2] G.. R. Krumpholz et al, "Power System Observability: A Practical Algorithm Using Network Topology", IEEE Trans. on PAS, Vol. 99, No.4, July/August 1980.

[3] K.A. Clements et al., "Power System State Estimation Residual Analysis: An Algorithm Using Network Topology", IEEE Trans. on PAS, Vol. 100, No.4, April 1981. 
[4] K.A. Clements et al, "Power System State Estimation with measurement Deficienvy: An Observability / Measurement Placement Algorithm", IEEE Trans. on PAS, Vol. 102, No.7, July 1983

[5] R. Tarjan, "Depth-First Search and Linear Graph Algorithms", SIAM Journal of Computers, Vol. 1, No.2, June 1972.

[6] T. L. Baldwin, "Power System Observability with Minimal Phasor Measurement Placement", IEEE Trans. in Power System, Vol.8, No.2, May 1993.

[7] T. W. Haynes et al, "Domination in Graphs Applied to Electric Power Networks", SIAM Journal of Discrete Math., vol. 15, No. 4, 2002.

[8] B. Mohammadi-Ivatloo, "Optimal Placement of PMUs for Power System Observability Using Topology Based
Formulated Algorithms", Journal of Applied Sciences, vol. 9, Issue 13, pp. 2463-2468, 2009

[9] Xingchen A. Yuan, “A Linear Algorithm for Minimum Phasor Measurement Units Placement",IEEE Tran.on Power System,2010.

[10] IEEE Work Group, "Synchronized Sampling and Phasor Measurements for relaying and Control", IEEE Trans. on Power Delivery, vol. 9, no. 1, January 1994, pp.442-449.

[11]S.Chakrabarti，E.kyriades，M.Albu "Uncertainity in power system state variables obtained through synchronized measurements" IEEE transactions on Instrumentations and Measurement,Vol. 58 No.8,Aug 2009,pp. 2452-2458. 